\title{
Recent lattice QCD results and phase diagram of strongly interacting matter
}

\author{
Heng-Tong Ding \\ Key Laboratory of Quark E Lepton Physics (MOE) and Institute of Particle Physics, \\ Central China Normal University, Wuhan 430079, China
}

\begin{abstract}
I review recent lattice QCD results on a few selected topics which are relevant to the heavy ion physics community. Special emphasis is put on the QCD equation of state at vanishing and nonzero baryon chemical potential, the onset of deconfinement of open strange and charmed hadrons, the contribution from experimentally not yet observed hadrons to the thermodynamics of the hadronic gas and their influence on freeze-out conditions of strange and light-quark hadrons.
\end{abstract}

Keywords:

Lattice QCD, heavy-ion collisions, Quark Gluon Plasma

\section{Introduction}

Lattice QCD is a discretized version of QCD in the Euclidean space time which reproduces QCD when the lattice spacing goes to zero, that is in the continuum limit. Most lattice QCD calculations which are at present available for use by the heavy ion community have been performed using non-chiral fermions which recover the flavor or chiral symmetry of QCD only in the continuum limit, e.g mostly used are staggered and Wilson fermions. Chiral fermions are generally much more expensive to work with. However, thanks to Moore's law, currently these actions are also used and start to produce interesting results on QCD thermodynamics, e.g. the value of the crossover temperature $T_{c}$ has been confirmed [1] and investigations of the restoration of $U(1)_{A}$ symmetry are also going on [2].

\section{Equation of state at vanishing and non-zero baryon density}

The equation of state (EoS) of QCD matter contains information on the change of degrees of freedom in different temperature as well as different baryon density regimes and reflects the transition between different states of the QCD matter. It is one of the important ingredients to understand the evolution of the fireball produced in heavy ion collisions through classical hydrodynamic equations. The computation of the QCD EoS has been one of the major goals in the lattice QCD community since 1980 [3].

Most of current EoS calculations are performed using the so-called staggered action. The shortfall of this action is that it breaks flavor symmetry at finite lattice spacing. The consequence of this is that in the staggered formulation of lattice QCD there are additional 15 heavier unphysical pions in addition to 1 physical Goldstone pion which lead to the physical pion spectrum only in the continuum limit. There are ways to improve this action at finite values of the lattice cutoff. One of them is the stout action, mostly used by Wuppertal-Budapest collaboration; another one is the Highly Improved Staggered Quark (HISQ) action which is mostly used by the HotQCD collaboration. At zero 

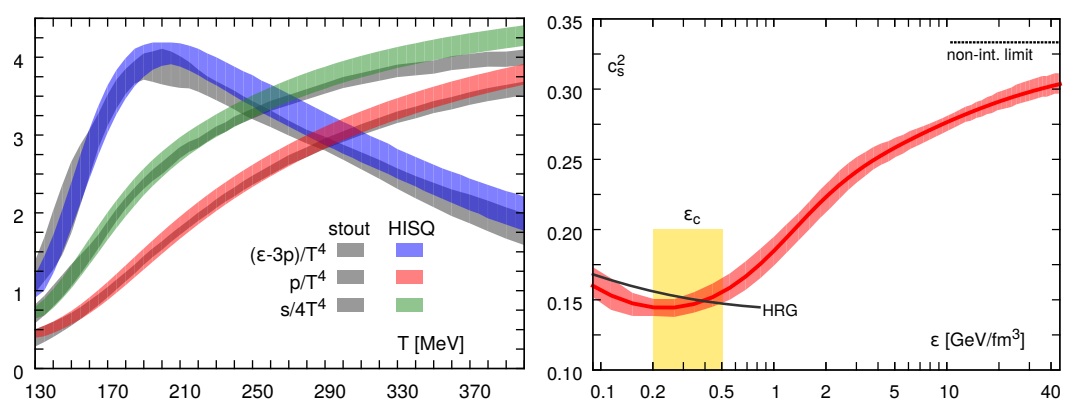

Figure 1. Left: Comparisons of continuum extrapolated results of trace anomaly $(\epsilon-3 p) / T^{4}$, pressure $p / T^{4}$ and entropy density $s / T^{4}$ from Wuppertal-Budapest (stout) [4] and HotQCD (HISQ) collaborations [5]. Right: The speed of sound squared from lattice QCD calculations using the HSIQ action and the HRG model as a function of energy density. Figures are taken from Ref. [5].

temperature the stout as well as the HISQ actions greatly reduce the masses of these 15 unwanted pions. Moreover, in the infinite temperature limit quantities calculated with the HISQ action, e.g. the pressure and energy density of the quark gluon plasma, approach their Stefan-Boltzmann limits faster than in calculations performed with the stout action at the same value of the lattice cutoff. However, continuum extrapolations of observables using either of these two actions can be safely performed on lattices at moderate values of the lattice cutoff.

In this conference the HotQCD collaboration has presented their recent results on the QCD EoS at vanishing baryon density [6]. Data with $N_{t}=4,6,8,10$ and 12 have been computed and $N_{t}=8,10$ and 12 have been used for the continuum extrapolation. Comparisons between results from the Wuppertal-Budapest (stout) and HotQCD (HISQ) collaborations are shown in the left panel of Fig. 1. It can be clearly seen that after the continuum extrapolations quantities obtained from stout and HISQ actions, i.e. trace anomaly $(\epsilon-3 p) / T^{4}$, pressure $p / T^{4}$ and entropy density $s / T^{4}$, are in good agreement in the temperature range from $130 \mathrm{MeV}$ to $400 \mathrm{MeV}$. A noticeable difference shown in the entropy density reaches about $7 \%$ at a temperature of $400 \mathrm{MeV}$. The resolution of this discrepancy requires more detailed calculations of the trace anomaly at higher temperatures. The right panel in Fig. 1] shows the speed of sound squared from lattice QCD calculations and the HRG model as a function of energy density. The chiral crossover temperature region, i.e. $T_{c} \in[149,163] \mathrm{MeV}$, is indicated by the yellow band in the plot. Here the value of the critical energy density $\epsilon_{c}$ lies in the range of $(180-500) \mathrm{MeV} / \mathrm{fm}^{3}$. At the softest point of the speed of sound squared the energy density is only slightly larger than that of normal nuclear matter, i.e. $\epsilon_{\text {nuclear }}=150 \mathrm{MeV} / \mathrm{fm}^{3}$. One may compare this energy density also to that inside a proton, $\epsilon_{\text {proton }}=450 \mathrm{MeV} / \mathrm{fm}^{3}$, and the energy density of an ideal quark gluon plasma in the crossover over temperature region, which ranges from $900 \mathrm{MeV} / \mathrm{fm}^{3}$ to $1440 \mathrm{MeV} / \mathrm{fm}^{3}$.
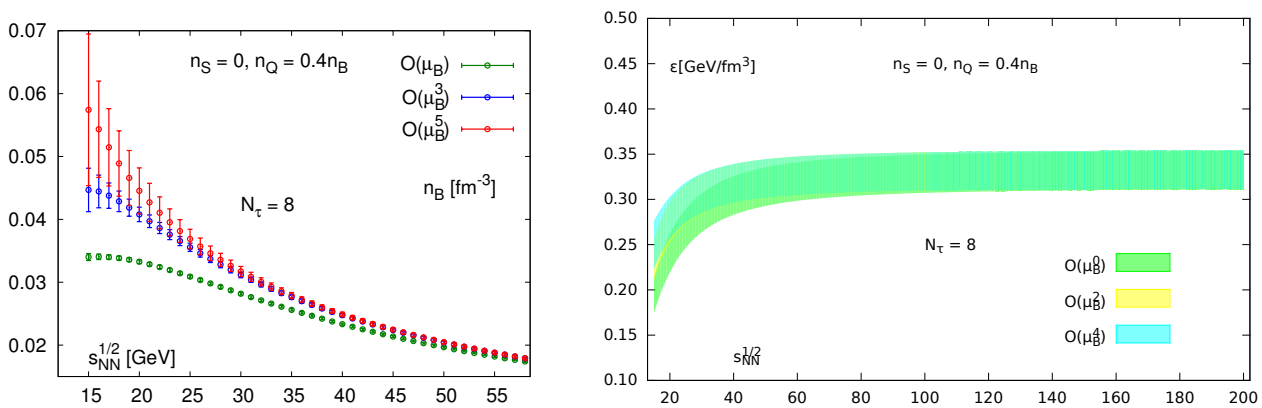

Figure 2. Baryon number density $n_{B}$ (left) and energy density $\epsilon$ (right) as function of $\sqrt{s_{N N}}$ along the freeze-out line. Calculations have been performed using the Taylor expansion method upto the order of $\mu_{B}^{5}$ and $\mu_{B}^{4}$, respectively. The freeze-out curve is obtained using the parameterization in Ref. [7]. Figures are taken from Ref. [8].

First results for the equation of state at nonzero baryon density obtained with the HISQ action in a Taylor series 
upto $4 \mathrm{~h}$ order have been reported by the BNL-Bielefeld-CCNU collaboration in this conference [8]. These calculations are performed on lattices with $N_{\tau}=6$ and 8 . The 2 nd order results from this study are consistent with those using the stout action [9]. As seen from the left panel of Fig. 2] a reliable description of baryon number density $n_{B}$ down to $\sqrt{s_{N N}} \sim 30 \mathrm{GeV}$ can be obtained by including terms up to $O\left(\mu_{B}^{3}\right)$; corrections from the next higher order, i.e. $O\left(\mu_{B}^{5}\right)$, reach about $30 \%$ at $\sqrt{s_{N N}} \sim 15 \mathrm{GeV}$. It is interesting to see in the right panel of Fig. 2 that the energy density $\epsilon$ stays almost constant, $\epsilon \sim 0.33 \mathrm{GeV} / \mathrm{fm}^{3}$, down to $\sqrt{s_{N N}} \sim 50 \mathrm{GeV}$. Note that at zero baryon chemical potential in the chiral crossover temperature region the critical energy density is $\epsilon_{c} \in[0.18,0.5] \mathrm{GeV} / \mathrm{fm}^{3}$.

\section{Deconfinement aspects of the QCD transition}

It is now well established that the QCD transition is just a rapid crossover at a transition temperature $T_{c} \sim 155$ $\mathrm{MeV}[10,11]$. Its chiral aspects manifest themselves in chiral quantities, such as the light quark number susceptibilities or the light quark chiral condensates, while the deconfinement aspects are related to the liberation of light degrees of freedom seen in the rapid change of the energy density or the change of heavier-quark degrees of freedom. As proposed long time ago by Matsui and Satz [12] quarkonium states can survive at temperatures above the critical temperature $T_{c}$. The fate of heavy quarkonium states has been studied extensively recently on the lattice [13]. Currently there are at least three approaches actively pursued to detect the modification of heavy quark bound states on the lattice. The first approach is to calculate the screening masses of a heavy quark-antiquark pair from spatial correlation functions. By comparing the screening masses at different values of the temperature with corresponding pole masses, i.e. the mass of quarkonium states in the vacuum, one can get some information on thermal modifications of bound states. The calculation of screening masses is straightforward and not expensive. However, the screening mass itself contains no more information other than the thermal modification of the pole mass [14]. Establishing a link to the melting of states is ambiguous. The second approach aims at the determination of the heavy quark potential from which spectral functions of quarkonium states can be obtained by using the Schrödinger equation. The imaginary and real parts of the heavy quark potential in general are related to the peak location and width of the spectral function of Wilson line correlation functions [15]. This approach is hampered by the noise to signal ratio of the Wilson line correlators which makes a precise determination of the width of the spectral function difficult. Recent progress has been made on developing an improved method to extract the spectral function from Wilson line correlators [16]. A third approach is to extract meson spectral functions from temporal Euclidean correlation functions. As the value of heavy quark mass $M$ is large the lattice spacing has to be small enough, i.e. $M a \ll 1$ to accommodate the heavy quark on the lattice. One way out is to directly calculate the heavy quarkonia correlation function by brute force, i.e. by reducing the lattice spacing to a sufficiently small value. Another way is to make use of effective theories, e.g. non-relativistic QCD, which however is appropriate only for bottom quarks and unfortunately does not allow to perform a continuum limit. Current state-of-the-art calculations, performed within quenched lattice QCD, suggests that all the charmonia states are melted at $T \geq 1.5 T_{c}$ [17]. Recent exploratory studies that also include dynamic quark degrees of freedom give consistent results [18]. For bottomonia it has recently been found that $S$ wave states start to melt at a temperature larger than $2 T_{c}$ while $P$ wave states melt already immediately above $T_{c}$ [19].

The fate of heavy-light mesons or baryons also reflects the change of the relevant degrees of freedom in strong interaction matter. For instance, the abundance of strange hadrons is considered as one of the signals that Quark Gluon Plasma is formed. In the heavy-light systems the net quantum number carried by the heavier quark, e.g. strangeness or charm, is nonzero rather than zero in the case of heavy quark-antiquark systems. As the electric charge $Q$ and baryon number $B$ of hadrons are integer quantum numbers in the confined hadronic phase but are fractional numbers in the deconfined QGP phase, fluctuations and correlations of these quantum numbers with strangeness or charm allow to probe the deconfinement of carriers of strangeness and charm degrees of freedom, i.e. the strange and charm quarks. These fluctuations are defined as the derivatives of pressure with respect to the chemical potential of a given quark flavor. In practice one needs to construct certain "order parameters" that vanish (approximately) in one phase and are large and nonzero in the other phase.

The starting point for such an analysis is the description of strangeness in an uncorrelated hadron gas. In the Hadron Resonance Gas (HRG) model, due to their large masses, heavy mesons and baryons follow Boltzmann statistics. The pressure of all the strange hadrons in an uncorrelated hadron resonance gas, $P_{S}^{H R G}$, can be decomposed into 
a mesonic, $P_{M}^{H R G}$, and baryonic part, $P_{B}^{H R G}[20]$

$$
P_{S}^{H R G}\left(\mu_{B}, \hat{\mu}_{S}\right)=P_{|S|=1, M}^{H R G} \cosh \left(\hat{\mu}_{S}\right)+\sum_{\ell=1}^{3} P_{|S|=i, B}^{H R G} \cosh \left(\hat{\mu}_{B}-\ell \hat{\mu}_{S}\right) .
$$

Fluctuations of conserved quantum numbers are obtained as derivatives of the pressure with respect to various chemical potentials $\hat{\mu}_{X}=\mu_{X} / T$, i.e. $\chi_{\mathrm{mn}}^{\mathrm{XY}}=\left.\frac{\partial^{(m+n)}\left(P\left(\hat{\mu}_{X}, \hat{\mu}_{Y}\right) / T^{4}\right)}{\partial \hat{\mu}_{X}^{m} \partial \hat{\mu}_{Y}^{n}}\right|_{\vec{\mu}=0}$ where $X, Y=B, Q, S, C$ and $\vec{\mu}=\left(\mu_{B}, \mu_{Q}, \mu_{S}, \mu_{C}\right)$ and $\chi_{0 n}^{X Y} \equiv \chi_{n}^{Y}$ and $\chi_{m 0}^{X Y} \equiv \chi_{m}^{X}$. Baryon-strangeness correlations that differ by an even number of derivatives with respect to $\mu_{B}$, are identical in a HRG. This is easily seen from the above equation. Combinations of conserved charge correlations such as $\chi_{31}^{\mathrm{BS}}-\chi_{11}^{B S}$ or $\chi_{2}^{B}-\chi_{4}^{B}$ thus vanish in an uncorrelated gas of hadrons within the classical Boltzmann approximation. Here $\chi_{31}^{\mathrm{BS}}-\chi_{11}^{B S}$ receive contributions only from strange hadrons while $\chi_{2}^{B}-\chi_{4}^{B}$ receive contributions from all hadrons. It is apparent from the left panel in Fig. 3 that all three quantities shown there would vanish in a HRG. They deviate from zero at almost the same temperature, i.e. in the vicinity of the chiral crossover temperature as indicated by the yellow band. This suggests that strange quark degrees of freedom start to get liberated from strange hadrons at almost the same temperature as light quarks. The whole analysis has been repeated using a different fermion action in Ref. [21].

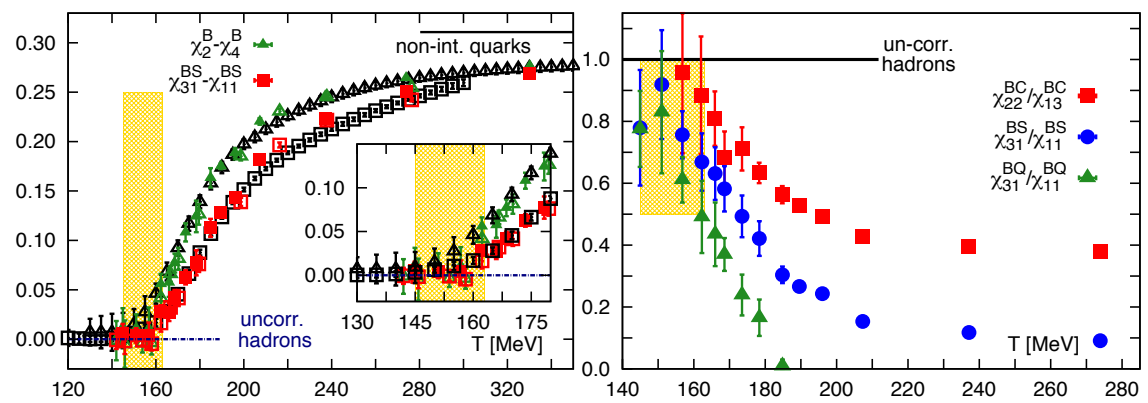

Figure 3. Left: Onset of the dissociation of open strange hadrons in the chiral crossover temperature region. Black points are results obtained using the stout action [21] while the other data points are those obtained using the HISQ action [20]. Right: Onset of open charm and open strange hadrons happen in the chiral crossover temperature region as seen from ratios of baryon-charm (BC), baryon-strangeness (BS) and baryon-electric charge (BQ) correlations [22]. Yellow bands shown in these two plots represent the temperature window of the chiral crossover $T_{c}=154 \pm 9 \mathrm{MeV}$.

The same concepts can also be applied to the open charm hadrons [22]. Since the charmed baryon sector with charm quantum number $C= \pm 1$ dominates the contribution to the partial pressure of all charmed baryons in an uncorrelated hadron resonance gas, $\chi_{n m}^{B C}$ with $n+m$ even and larger than 2 is approximately equal to $\chi_{11}^{B C}$. Thus three quantities, $\chi_{22}^{B C} / \chi_{13}^{B C}, \chi_{31}^{B S} / \chi_{11}^{B S}$ and $\chi_{31}^{B Q} / \chi_{11}^{B Q}$, which receive contributions only from the charm, strange and (dominantly) light quark sectors, respectively, should be equal to unity as long as an uncorrelated hadron resonance gas model provides an appropriate description of the thermodynamics of the medium. It is obvious from the right panel in Fig. 3 that all three quantities start to deviate from unity in the chiral crossover region. This indicates that a description in terms of a HRG model breaks down for baryonic correlations involving light, strange, or charm quarks, i.e. open charm/strange hadrons start to dissociate in or just above the chiral crossover region.

\section{Thermodynamic contributions from unobserved hadrons near QCD transition}

A hadron resonance gas model that approximates QCD should include all states that are predicted by Quantum Chromodynamics. However, there are quite a few states that are predicted in relativistic Quark Model (QM) and lattice QCD calculations [23] that have not yet been observed in experiments [24] and thus do not show up in the particle data tables. It thus is interesting to see whether these additional states can have any significant contribution to QCD thermodynamics [22, 25].

One can check the partial pressures of open charm and strange hadrons in HRG models based on a particle spectrum predicted in QM models (QM-HRG) and the spectrum listed in the PDG table (PDG-HRG). It turns out 
that there are only small difference in partial meson pressures while differences in baryon sector are much larger. This simply reflects the fact that the experimental knowledge of the strange and charm meson spectrum is more complete than that for baryons. The difference is more pronounced in the ratio of partial pressures of baryons and mesons [22, 25]. In this spirit one can also construct observables that reflect the ratio of partial pressures of charmed baryons and mesons. For instance, quantities like $\chi_{13}^{B C} /\left(\chi_{4}^{C}-\chi_{13}^{B C}\right), \chi_{112}^{B Q C} /\left(\chi_{13}^{Q C}-\chi_{112}^{B Q C}\right)$ and $-\chi_{112}^{B S C} /\left(\chi_{13}^{S C}-\chi_{112}^{B S C}\right)$ give the relative contributions of charmed baryons to open charm mesons, charged-charmed baryons to open charm charged mesons and strange-charmed baryons to strange-charmed mesons, respectively. As seen in the left panel of Fig. 4 the temperature dependence of these three quantities in the chiral crossover region can be better described by the solid line (QM-HRG), i.e. results obtained from an HRG model using the particle spectrum predicted in the QM model. The popularly used HRG model based on the particle spectrum listed in the PDG table, i.e. PDG-HRG shown in the plot, fails to describe the lattice data. The right panel in Fig. 4 shows similar quantities for open strange hadrons: $-\chi_{11}^{B S} / \chi_{2}^{S}$ and $B_{i}^{S} / M_{i}^{S}$ reflect the relative contributions of strange baryons to open strange mesons in strange-baryon correlations and in partial pressures, respectively. The same conclusion can be drawn from this plot as for the charm sector. The QM-HRG agrees better with the lattice data than the PDG-HRG. This provides clear evidence for contributions from non-PDG listed hadrons to QCD thermodynamics and the transition from hadronic matter to the Quark Gluon Plasma. The importance of these additional states has also been pointed out in Ref. [26]. An important consequence of these states for the determination of freeze-out conditions for strange hadrons will be discussed in the next section.

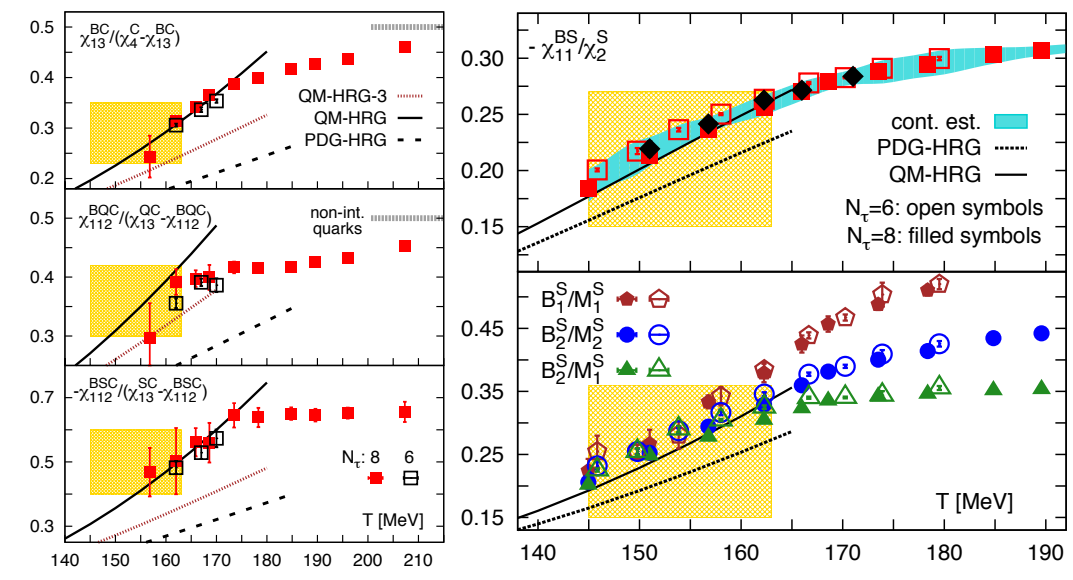

Figure 4. Observation of thermodynamic contributions from open charm (left) and open strange (right) hadrons that are predicted in the relativistic Quark Model (QM) but not listed in the PDG table. Figures are taken from Ref. [22] and Ref. [25], respectively. Yellow bands shown in these two plots represent the temperature window of the chiral crossover $T_{c}=154 \pm 9 \mathrm{MeV}$.

\section{Freeze-out conditions}

It has been proposed in Ref. [27] that the freeze-out/hadronization conditions can be determined by comparing fluctuations of net conserved charges calculated on the lattice to the corresponding experimental observables. For instance the mean value, variance and skewness of net electric charge can be directly related to cumulants of net electric charge fluctuations which are just the derivatives of pressure with respect to charge chemical potentials. They can be calculated on the lattice in a straightforward way. These quantities can also be measured in heavy ion experiments with a certain precision.

The ratio $R_{31}^{Q}=S_{Q} \sigma_{Q}^{3} / M_{Q}$ is related to skewness $S_{Q}$, variance $\sigma_{Q}$ and mean value $M_{Q}$ of net electric charge distributions that are accessible in experimental measurements. The left panel in Fig. 5 shows that $R_{31}^{Q}$ has only a weak dependence on the baryon density but changes rapidly with temperature. It thus can be used as a thermometer to extract the freeze-out temperatures of hadrons. On the other hand, the observable $R_{12}^{Q}=M_{Q} / \sigma_{Q}^{2}$ has a strong dependence on $\mu_{B}$ and it can be used as a baryometer to extract the baryon chemical potential at freeze-out. The approach to the determination of freeze-out conditions has also been pursued by using a different disrectization scheme on the lattice 

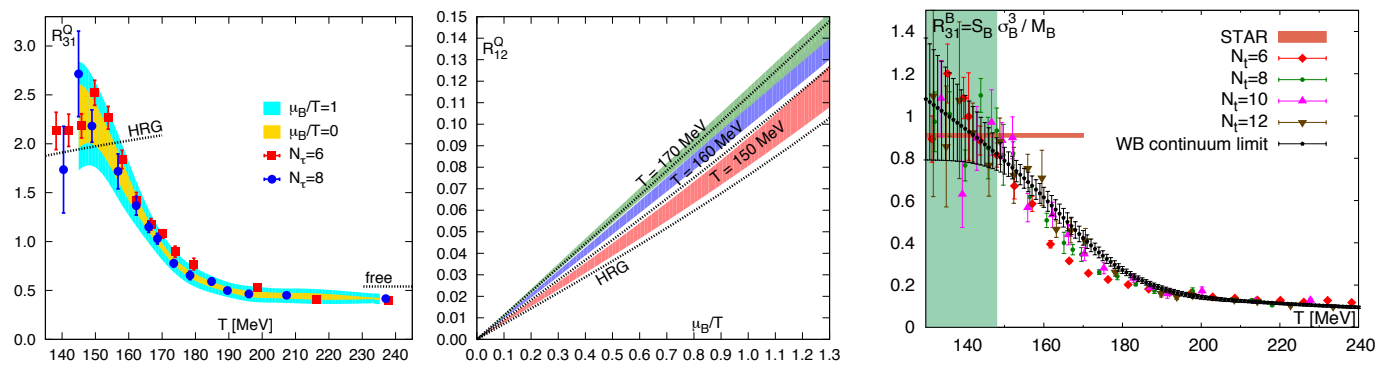

Figure 5. The ratios $R_{31}^{Q}=S_{Q} \sigma_{Q}^{3} / M_{Q}$ versus temperature for $\mu_{B}=0$ (left) and $R_{12}^{Q}=M_{Q} / \sigma_{Q}^{2}$ versus $\mu_{B} / T$ for three values of temperatures (middle) calculated on the lattice [27]. Ratios $R_{31}^{Q}$ and $R_{12}^{Q}$ can serve as thermometer to determine the temperature and baryometer to determine the baryon chemical potential at the freeze-out, respectively. The right hand panel shows a comparison of lattice QCD data with the STAR measurements on the $S_{B} \sigma_{B}^{3} / M_{B}$ and the upper limit of freeze-out temperature $T_{f}^{\max }=148 \pm 4 \mathrm{MeV}$ is obtained from the comparison [33].

later on [28]. However, current experimental data for net electrical charge fluctuations have large errors and at present do not allow to perform a detailed comparison with lattice QCD data [29, 30]. To arrive at a better comparison of net charge fluctuation higher precision data are certainly needed from e.g. BES-II [31]. More accurate data are available on net proton number fluctuations [32]. In the right panel of Fig. 5] a comparison of lattice calculations of net baryon number fluctuations and experimental measurements of net proton number fluctuations is shown. This provides an upper bound on the freeze-out temperature, i.e. $T_{f} \leq 148 \pm 4 \mathrm{MeV}$ [33]. However, one needs to keep in mind that the net baryon and net proton fluctuations are not the same [34].
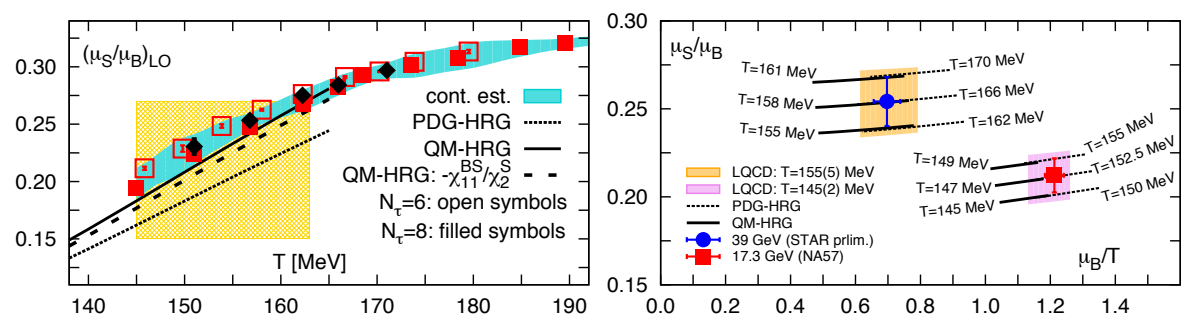

Figure 6. Left: Imprints of contributions from unobserved strange hadrons to $-\chi_{11}^{B S} / \chi_{2}^{S}$ in $\mu_{S} / \mu_{B}$. Right: Comparison of freeze-out temperature obtained from lattice QCD calculations (LQCD) and Hadron Resonance Gas model using the particle spectrum listed in the PDG table (PDG-HRG) and predicted from Quark Model (QM-HRG) by reproducing the relation of $\mu_{S} / \mu_{B}$ to $\mu_{B} / T$ determined from STAR and NA57 experiments. The temperatures obtained from QM-HRG are consistent with those from LQCD and are always smaller than those from PDG-HRG by around 5 8 MeV. Figures are taken from Ref. [20].

One interesting question that has been discussed frequently in the recent literatures is whether strange hadrons freeze out at a higher temperature than light-quark hadrons [21, 35]. As shown in Fig. 3 and also in investigations of the screening masses of strange mesons [14], strange hadrons start to get deconfined in the same temperature window as light-quark hadrons do. This suggests that they will probably hadronize or freeze out at almost the same temperature. On the other hand, the indication of a higher freeze-out temperature for strange hadrons found in Ref. [35] is mainly based on the quality of fits to the experimental data using the PDG-HRG model, i.e. fits become better when different freeze-out temperatures are assumed or certain particle ratios are left out from the fits.

In this context it is important to understand systematic effects that may arise from thermodynamic contributions of additional, unobserved strange hadrons. Strangeness neutrality in heavy ion collisions enforces a dependence of the strangeness chemical potential $\mu_{S}$ on baryon chemical potential $\mu_{B}$ and temperature $T$. To leading order $\mu_{S}$ can then be expanded in a Taylor series of $\mu_{B}$ as follows [20]

$$
\frac{\mu_{S}}{\mu_{B}} \simeq-\frac{\chi_{11}^{B S}}{\chi_{2}^{S}}-\frac{\chi_{11}^{Q S}}{\chi_{2}^{S}} \frac{\mu_{Q}}{\mu_{B}}+O\left(\mu_{B}^{2}\right) .
$$


In this equation the term $-\chi_{11}^{B S} / \chi_{2}^{S}$ probes the relative abundance of strange baryons to open strange mesons. The sensitivity to the hadron spectrum is evident from the right panel of Fig. 4 At a given value of the temperature the value of $\mu_{S} / \mu_{B}$ therefore is influenced by thermodynamic contributions that arise from additional strange hadrons. It can be clearly seen in the left panel of Fig. 6 that the contribution from the term $-\chi_{11}^{B S} / \chi_{2}^{S}$ dominates in $\mu_{S} / \mu_{B}$ and that the calculation based on QM-HRG describes the lattice QCD results much better than a PDG-HRG calculation. Consequently, at a fixed value of $\mu_{S} / \mu_{B}$ a smaller value of temperature will be extracted when using lattice QCD and QM-HRG model calculations than by using PDG-HRG model calculations.

The relation of $\mu_{S} / \mu_{B}$ with $\mu_{B} / T$ can be obtained from a two-parameter fit to ratios of yields of strange anti-baryons to baryons using the following ansatz motivated from the HRG model [20]:

$$
R_{H} \equiv \frac{\bar{H}_{S}}{H_{S}}=\exp \left[-2\left(\mu_{B}^{f} / T^{f}\right) \times\left(1-\left(\mu_{S}^{f} / \mu_{B}^{f}\right)|S|\right)\right],
$$

where $|S|$ is the absolute value of strangeness carried by the strange baryons. Since details of hadron spectrum cancel in the ratio $R_{H}$, the ansatz is valid for both PDG-HRG and QM-HRG. It allows to extract two fitting parameters $\mu_{B}^{f} / T^{f}$ and $\mu_{S}^{f} / \mu_{B}^{f}$. Thus the freeze-out temperature $T^{f}$ of strange hadrons can be obtained by adjusting the value of temperature in lattice QCD and HRG model calculations to reproduce values of $\mu_{S}^{f} / \mu_{B}^{f}$ and $\mu_{B}^{f} / T^{f}$ obtained from fits to experimental data. The right panel of Fig. 6 shows the reproduction of the value of $\mu_{S}^{f} / \mu_{B}^{f}$ and $\mu_{B}^{f} / T^{f}$ by adjusting the value of temperature in the lattice QCD calculations and PDG-HRG as well as QM-HRG model calculations. The resulting value of $T^{f}$ from QM-HRG model calculations is consistent with that from lattice QCD calculations, however, it is smaller than that from PDG-HRG model calculations by around 5-8 MeV. This is mainly due to the fact that the presence of additional, experimentally still unobserved states, gets imprinted in $\mu_{S}^{f} / \mu_{B}^{f}$ as shown in Eq. 2 and the left panel of Fig. 6 . The freeze-out temperature $T^{f}$ determined for strange hadrons is thus is smaller by about 5-8 $\mathrm{MeV}$ when experimentally yet unobserved strange hadrons are taken into account. It becomes similar to the freeze-out temperature of light-quark hadrons. In other words there maybe no flavor hierarchy in the freeze-out conditions and light-quark and strange hadrons may freeze out in the same temperature region.

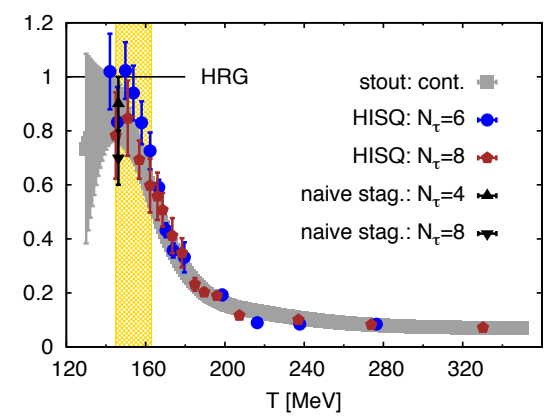

Figure 7. $\chi_{B}^{4} / \chi_{B}^{2}$ as a function of temperature at $\mu_{B}=0$ obtained using the stout [28], HISQ [20] and naïve staggered [36] actions.

\section{Towards the critical point}

To understand the dip shown in the experimental data of kurtosis of baryon number [32] in terms of properties of conserved charge fluctuations calculated on the lattice, one can expand kurtosis in a Taylor series of $\mu_{B}$ as follows

$$
\left(\kappa \sigma^{2}\right)_{B}=\frac{\chi_{4, \mu}^{B}}{\chi_{2, \mu}^{B}}=\frac{\chi_{4}^{B}}{\chi_{2}^{B}}\left[1+\left(\frac{\chi_{6}^{B}}{\chi_{4}^{B}}-\frac{\chi_{4}^{B}}{\chi_{2}^{B}}\right)\left(\frac{\mu_{B}}{T}\right)^{2}+\cdots\right] .
$$

The curvature of the kurtosis is then controlled by the difference of $\chi_{6}^{B} / \chi_{4}^{B}$ and $\chi_{4}^{B} / \chi_{2}^{B}$ up to the second order of $\mu_{B} / T$. A general agreement on the temperature dependence of $\chi_{4}^{B} / \chi_{2}^{B}$ is reached from lattice QCD calculations using different 
actions. A determination of the 6th order cumulants still is missing and is crucially needed. Recent progress has also been made to directly simulate the QCD phase diagram at nonzero baryon chemical potential using the Complex Langevin method [37].

\section{Summary}

Since the last Quark Matter conference considerable progress has been made in understanding the equilibrium thermodynamics of strong interaction matter through lattice QCD calculations. Some highlights are: A consensus has been reached on the QCD Equation of State at vanishing baryon density by the HotQCD and Wuppertal-Budapest collaborations. The QCD EoS at nonzero baryon chemical potential, which is important for the BES program, has been calculated using Taylor expansions up to fourth order in $\mu_{B} / T$. The baryon number density can be reliably obtained in $O\left(\mu_{B}^{3}\right)$ to describe heavy ion collisions down to $\sqrt{s_{N N}} \sim 30 \mathrm{GeV}$; The energy density at freeze-out remains roughly constant down to $\sqrt{s_{N N}} \sim 50 \mathrm{GeV}$. By looking at the correlations between charm or strangeness quantum numbers with baryon or electrical charge quantum numbers the onset of the melting of open charm and strange hadrons can be identified. In both cases it happens in the chiral crossover temperature region. Evidence has been found for the thermodynamic relevance of experimentally yet unobserved strange hadrons. They may influence the determination of freeze-out conditions from experimentally measured particle yields.

\section{References}

[1] T. Bhattacharya, M. I. Buchoff, N. H. Christ, H. -T. Ding, R. Gupta, C. Jung, F. Karsch and Z. Lin et al., arXiv:1402.5175 [hep-lat].

[2] M. I. Buchoff, M. Cheng, N. H. Christ, H. -T. Ding, C. Jung, F. Karsch, Z. Lin and R. D. Mawhinney et al., Phys. Rev. D 89 (2014) 054514, A. Bazavov et al. [HotQCD Collaboration], Phys. Rev. D 86 (2012) 094503, G. Cossu et al., Phys. Rev. D 87 (2013) $11,114514$.

[3] J. Engels, F. Karsch, H. Satz and I. Montvay, Phys. Lett. B 101 (1981) 89.

[4] S. Borsanyi, Z. Fodor, C. Hoelbling, S. D. Katz, S. Krieg and K. K. Szabo, Phys. Lett. B 730 (2014) 99 |arXiv:1309.5258 [hep-lat]].

[5] A. Bazavov, T. Bhattacharya, C. DeTar, H. -T. Ding, S. Gottlieb, R. Gupta, P. Hegde and U. M. Heller et al., arXiv:1407.6387 [hep-lat].

[6] A. Bazavov, this proceedings.

[7] J. Cleymans and K. Redlich, Phys. Rev. C 60 (1999) 054908.

[8] P. Hegde, this proceedings.

[9] S. Borsanyi, G. Endrodi, Z. Fodor, S. D. Katz, S. Krieg, C. Ratti and K. K. Szabo, JHEP 1208 (2012) 053.

[10] S. Borsanyi et al. [Wuppertal-Budapest Collaboration], JHEP 1009(2010) 073.

[11] A. Bazavov, T. Bhattacharya, M. Cheng, C. DeTar, H. T. Ding, S. Gottlieb, R. Gupta and P. Hegde et al., Phys. Rev. D 85 (2012) 054503.

[12] T. Matsui and H. Satz, Phys. Lett. B 178 (1986) 416.

[13] See e.g. H. T. Ding, arXiv:1404.5134[hep-lat] and references therein.

[14] Y. Maezawa et al., arXiv:1312.4375 F. Karsch el al., Phys. Rev. D 85 (2012) 114501, M. Cheng et al., Eur. Phys. J. C 71 (2011) 1564.

[15] Y. Burnier and A. Rothkopf, Phys. Rev. D 86 (2012) 051503 [arXiv:1208.1899 [hep-ph]].

[16] Y. Burnier and A. Rothkopf, Phys. Rev. Lett. 111 (2013) 18, 182003 |arXiv:1307.6106 [hep-lat]].

[17] H. -T. Ding, A. Francis, O. Kaczmarek, F. Karsch, H. Satz and W. Soeldner, Phys. Rev. D 86 (2012) 014509, H. Ohno, arXiv:1311.4565

[18] S. Borsanyi, S. Drr, Z. Fodor, C. Hoelbling, S. D. Katz, S. Krieg, S. Mages and D. Nogradi et al., JHEP 1404 (2014) 132.

[19] G. Aarts et al., arXiv:1402.6210 [hep-lat], JHEP 1312 (2013) 064, JHEP 1111 (2011) 103.

[20] A. Bazavov, H. -T. Ding, P. Hegde et al., Phys. Rev. Lett. 111, 082301 (2013).

[21] R. Bellwied, S. Borsanyi, Z. Fodor, S. D. Katz and C. Ratti, Phys. Rev. Lett. 111 (2013) 202302.

[22] A. Bazavov, H. -T. Ding, P. Hegde et al., arXiv:1404.4043 [hep-lat], S. Sharma, arXiv:1408.2332 [hep-lat], this proceedings.

[23] See e.g. M. Padmanath, R. G. Edwards, N. Mathur and M. Peardon, arXiv:1311.4806 [hep-lat].

[24] See e.g. D. Ebert, R. N. Faustov and V. O. Galkin, Eur. Phys. J. C 66 (2010) 197, Phys. Rev. D 84 (2011) 014025.

[25] A. Bazavov, H.-T. Ding, P. Hegde et al., Phys. Rev. Lett. 113 (2014) 072001 [arXiv:1404.6511 [hep-lat]], C. Schmidt, this proceedings.

[26] A. Majumder and B. Muller, Phys. Rev. Lett. 105 (2010) 252002, M. Beitel, K. Gallmeister and C. Greiner, arXiv:1402.1458 [hep-ph].

[27] A. Bazavov, H. T. Ding, P. Hegde et al., Phys. Rev. Lett. 109 (2012) 192302.

[28] S. Borsanyi, Z. Fodor, S. D. Katz, S. Krieg, C. Ratti and K. K. Szabo, Phys. Rev. Lett. 111 (2013) 062005.

[29] S. Mukherjee and M. Wagner, PoS CPOD 2013 (2013) 039 [arXiv:1307.6255[nucl-th]].

[30] L. Adamczyk et al. [STAR Collaboration], arXiv:1402.1558 [nucl-ex].

[31] G. Odyniec, J. Phys. Conf. Ser. 455 (2013) 012037.

[32] L. Adamczyk et al. [STAR Collaboration], Phys. Rev. Lett. 112 (2014) 162301

[33] S. Borsanyi, Z. Fodor, S. D. Katz, S. Krieg, C. Ratti and K. K. Szabo, Phys. Rev. Lett. 113 (2014) 052301, C. Ratti, this proceedings

[34] M. Kitazawa, this proceedings.

[35] P. Alba et al., arXiv:1403.4903 [hep-ph], K. A. Bugaev et al., Europhys. Lett. 104 (2013) 22002, S. Chatterjee, R. M. Godbole and S. Gupta, Phys. Lett. B 727 (2013) 554, A. Andronic, P. Braun-Munzinger, K. Redlich and J. Stachel, Nucl. Phys. A $904-905$ (2013) 535c.

[36] S. Gupta, N. Karthik and P. Majumdar, Phys. Rev. D 90 (2014) 034001 arXiv:1405.2206 [hep-lat]].

[37] D. Sexty, this proceedings. 